\title{
Understanding Post-Prandial Hyperglycemia in Patients with Type 1 and Type 2 Diabetes: A Web-based Survey in Germany, the UK, and USA
}

\author{
Meryl Brod $\cdot$ Annie Nikolajsen $\cdot$ James Weatherall $\cdot$ Kathryn M. Pfeiffer
}

Received: April 4, 2016/ Published online: May 27, 2016

(C) The Author(s) 2016. This article is published with open access at Springerlink.com

\begin{abstract}
Introduction: To explore how patients with diabetes experience post-prandial hyperglycemia (PPH) or elevated blood glucose (BG) following a meal.
\end{abstract}

Methods: A web-based survey of patients with type 1 or type 2 diabetes using bolus insulin in Germany, the USA, and the UK was conducted. Results: A total of 906 respondents completed the survey. PPH was a frequent occurrence among patients with type 1 and type 2 diabetes; $61.9 \%$ of respondents had

Enhanced content To view enhanced content for this article go to http://www.medengine.com/Redeem/ D4D4F0603967A69F.

M. Brod ( $\square)$

The Brod Group, 219 Julia Avenue, Mill Valley, CA 94941, USA

e-mail: mbrod@thebrodgroup.net

A. Nikolajsen

Market Access, Payer Engagement, Novo Nordisk

A/S, Vandtårnsvej 114, 2860 Søborg, Denmark

J. Weatherall

Health Economic Outcomes Research,

Novo Nordisk, Inc., 800 Scudders Mill Road,

Plainsboro, NJ, USA

K. M. Pfeiffer

Health Outcomes Research, The Brod Group,

219 Julia Avenue, Mill Valley, CA, USA experienced PPH in the past week, and differences by diabetes type were not significant. More than half of the respondents reported that they knew they were experiencing PPH because they had measured their BG $(64.8 \%)$ and/or because they “just didn't feel right" (51.9\%). The most frequently reported reasons given for $\mathrm{PPH}$ were eating more fat/sugar than estimated (31.2\%) and over-eating in terms of their calculated bolus insulin dose (30.4\%). The most common situations/factors contributing to $\mathrm{PPH}$ were stress (27.4\%), eating at a restaurant (24.9\%), being busy (21.1\%), and/or feeling tired (19.2\%). The most frequent corrective actions respondents took following $\mathrm{PPH}$ were testing BG and taking bolus insulin based on the reading $(62.0 \%)$, and/or eating less/more carefully at their next meal or snack (18.8\%). Additionally, significant differences in the reasons and contributing factors given for $\mathrm{PPH}$ and corrective actions following PPH, as well as emotions experienced when taking bolus insulin, were found by diabetes type.

Conclusion: These findings shed light on how patients with diabetes experience and manage PPH on a day-to-day basis and have 
implications for improving diabetes self-management. Clinicians and diabetes educators should help patients address eating habits and lifestyle issues that may contribute to PPH.

Funding: This study was sponsored by Novo Nordisk.

Keywords: Disease management; Health survey; Patient outcome assessment; Post-prandial hyperglycemia; Type 1 diabetes; Type 2 diabetes

\section{INTRODUCTION}

Glycemic control is an important part of diabetes self-management for patients with type 1 and type 2 diabetes [1]. Research suggests that adequate and timely glycemic control, as measured by hemoglobin A1c (HbA1c) values, reduces the risks of microvascular and macrovascular complications related to diabetes [1-4]. While research on diabetes management has focused primarily on the role of HbA1c and fasting plasma glucose (FPG) for the measurement of glycemic control, recent data suggest that blood glucose (BG) after eating or post-prandial blood glucose (PPG), also plays an important role in glycemic control and glycemic variability on a day-to-day basis, and provide additional information not captured by HbA1c values [5]. In particular, elevated PPG or post-prandial hyperglycemia (PPH) may be an important consideration for improving glycemic control and diabetes self-management, and ultimately reduce the risk of diabetes-related complications.

Currently, there is no consensus on the definition of $\mathrm{PPH}$ in the research literature or among clinical communities. The American Diabetes Association (ADA), the International
Diabetes Federation (IDF), and the American Association of Clinical Endocrinologists (AACE), for instance, use different PPG targets in their guidelines for clinicians and patients. According to the ADA, the PPG target $1-2 \mathrm{~h}$ post-meal is $<180 \mathrm{mg} / \mathrm{dL}$, while the IDF recommends a PPG target of $<160 \mathrm{mg} / \mathrm{dL}$; the AACE's recommended PPG target $2 \mathrm{~h}$ post-meal is $<140 \mathrm{mg} / \mathrm{dL}[1,2,6]$. A recent clinical study based on the IDF threshold found that among patients with type 2 diabetes not treated with insulin therapy, $84 \%$ had experienced $\mathrm{PPH}$ at least once in the previous week [7].

Post-prandial blood glucose (PPG) is an important part of overall glycemic control, and research has shown that $\mathrm{PPH}$ is associated with a significantly increased risk of cardiovascular disease (CVD) and cardiovascular events among individuals with and those without diabetes [8-10]. Some research suggests that PPG may be a better predictor of cardiovascular events than FPG [11, 12]. In addition to CVD and cardiovascular events, several long-term studies of individuals with and without diabetes have shown that PPH or elevated post-challenge glucose following an oral glucose tolerance test is significantly associated with increased risks of both all-cause mortality and CVD mortality $[11,13-16]$, as well as pancreatic cancer mortality [17]. PPH has also been associated with a number of other negative health outcomes, including decreased cognitive functioning among elderly patients with type 2 diabetes [18], oxidative stress, endothelial dysfunction, retinopathy, and some cancers [2]. Research has also suggested that PPH may contribute to greater use of healthcare resources and related costs among diabetic patients [19]. Further, PPG control may be particularly challenging for diabetic patients who require more complex insulin regimens, comprising, for example, a combination of basal and bolus insulins. There is 
evidence that patients with type 1 diabetes treated with basal and bolus insulin face challenges in calculating and adjusting their insulin doses over time, and that they may rely on bolus insulin as a "correction" following $\mathrm{PPH}$, rather than examining BG measurement patterns over time and adjusting insulin doses accordingly to improve overall BG control [20].

Despite the importance of PPH for diabetes self-management, relatively little is known about how patients with type 1 and type 2 diabetes experience PPH in their daily lives and how $\mathrm{PPH}$ contributes to the complexities of diabetes self-management. We conducted a web-based survey to better understand how patients with type 1 and type 2 diabetes experience $\mathrm{PPH}$ and its role in diabetes self-management.

\section{METHODS}

A web-based survey of patients with type 1 or type 2 diabetes who self-administered bolus insulin therapy was conducted in Germany, the United Kingdom (UK), and the United States (USA), from July to November 2013. The study received ethics approval from the Copernicus Group Institutional Review Board (Tracking \# TBG1-11-116) prior to commencement. All procedures followed were in accordance with the ethical standards of the responsible committee on human experimentation (institutional and national) and with the Helsinki Declaration of 1964, as revised in 2013. Informed consent was obtained from all patients included in the study.

\section{Survey Development}

To develop the survey, semi-structured focus groups were conducted with 77 participants recruited by focus group facilities who were diagnosed with diabetes and using bolus insulin in Germany $(n=20)$, the UK $(n=17)$, and the USA $(n=40)$. Survey items were generated through the analysis of focus group transcripts, based on adapted grounded theory [21]. Once generated, survey items underwent cognitive debriefing with 12 individuals diagnosed with diabetes (recruited by focus group facilities, but not participants in the study focus groups) to reach consensus that instructions and items were clear, understandable, relevant and inoffensive, to ensure the recall period was appropriate, and to confirm that the format and structure of the survey were acceptable. The final survey comprised 85 questions and took approximately $30 \mathrm{~min}$ to complete.

\section{Participants}

The web-based survey was administered to participants recruited from research panels maintained by professional market research firms in the three countries via a secure internet server, which participants accessed via their computers. Potential respondents were selected from pre-existing panels of individuals who had previously agreed to be approached to participate in surveys. These pre-existing panels were used for research purposes only. Respondents were required to complete a brief online screening survey to ensure eligibility for participation. During screening, panel participants were not aware that a diagnosis of diabetes was required for survey eligibility; participants were also asked about a number of other conditions they may have been diagnosed with to reduce the risk of potential misreporting of a diabetes diagnosis to participate in the survey. Minimal incentives were provided for panel participation. 
To be eligible for the survey, respondents were required to meet the following criteria: aged 18 years or older, diagnosed with type 1 or type 2 diabetes by a physician or healthcare professional, prescribed self-administered bolus insulin therapy and not using pre-mixed insulin or glucagon-like peptide-1 analogs. Use of oral antidiabetic medication was permitted if required. Based on the inclusion and exclusion criteria, approximately $7 \%$ of panel participants who were approached for the survey were eligible to participate. Recruitment quotas were established for each country for the method of insulin administration ( $\sim 20 \%$ pump users and $\sim 80 \%$ injection), diabetes type ( $\sim 40 \%$ type 1 and $\sim 60 \%$ type 2 ), age ( $\sim 40 \%, \quad 18-30$ years; $\sim 40 \%, \quad 31-60$ years; $\sim 20 \%$, over 60 years), and work status ( $\sim 50 \%$ employed) to ensure adequate sampling across groups and generalizability of results. Respondents received a modest honorarium (approximately \$15 USD) for completing the survey.

\section{Survey Variables}

All responses to the survey were self-reported. The survey items assessed the following: demographic and diabetes health status, PPG management and experience of $\mathrm{PPH}$, emotions experienced when using bolus insulin, and diabetes-related concerns.

Demographic variables included country, age, gender, marital status, education level, employment status, and number of hours worked per week. Diabetes health status characteristics included age at diagnosis, diabetes type, age at onset of insulin therapy, method of insulin administration, respondents' perceptions of how well their diabetes was controlled, and respondents' perceptions of their general health status. Dietary variables were also assessed including the number of skipped meals in the previous week and whether the respondent ate at a restaurant, ate takeout food, and/or had eaten at a friend's home in the past week. Physical activity was also reported.

PPG/PPH and diabetes self-management variables were respondent-reported based on their experience of out-of-range BG after eating in the past week (7 days). Measures included the number of episodes of PPH and episodes of post-prandial hypoglycemia in the past week. Dichotomous variables indicating experience of $\mathrm{PPH}$ and post-prandial hypoglycemia in the past week were also created. Respondents also reported on their most recent episode of out-of-range PPG and the number of days since this last incident. Respondents whose most recent experience of out-of-range PPG was PPH (as opposed to post-prandial hypoglycemia or being unsure) were asked additional questions about their experiences of $\mathrm{PPH}$, including how they knew they were experiencing $\mathrm{PPH}$, reasons for $\mathrm{PPH}$, situations and factors contributing to $\mathrm{PPH}$, and corrective actions taken following PPH.

Respondents were questioned on the emotions they experienced when taking bolus insulin doses (e.g., empowered, grateful, worried, discouraged) and their level of confidence in calculating bolus insulin doses accurately. Respondents also reported on general diabetes-related concerns, indicating how worried they were about using their prescribed insulin device, hypoglycemic/hyperglycemic events, future diabetes complications, whether insulin was controlling their diabetes, whether they were able to calculate bolus doses correctly, and problems contacting their healthcare provider. 


\section{Data Analysis}

The sample size was chosen based on the analysis plan and to ensure that comparisons could be made across groups. Analyses of the survey data included descriptive statistics (means, standard deviations, percentages, and ranges) and measures of association (comparison of means and cross-tabulations). Statistical significance tests were conducted based on the type of analysis: ANOVA was used for comparison of means among three groups, and t tests were used for comparison of means between two groups. Pearson's Chi square was used to test for significant associations between categorical variables. Experience of $\mathrm{PPH}$ was self-reported. The number of episodes of PPH and episodes of post-prandial hypoglycemia in the past week were both trimmed at the top 5\% level to correct for potential over-reporting errors. Respondent experiences of $\mathrm{PPH}$, including contributing factors and responses to $\mathrm{PPH}$, were based on questions asked of respondents whose most recent out-of-range PPG episode was PPH. Respondents with and without PPH in the previous week were also compared in terms of health and diabetes characteristics, emotions when taking bolus insulin, diabetes-related concerns, and diabetes self-management. All analyses were conducted by country and by diabetes type. IBM SPSS Statistics version 22 (IBM, New York, USA) was used for all data analyses.

\section{RESULTS}

\section{Sample Description}

A total of 906 respondents in three countries completed the survey [Germany, $n=305$ (type
1, $n=130$; type 2, $n=175$ ); UK, $n=236$ (type 1 , $n=117$; type $2, n=119$ ), US, $n=365$ (type 1 , $n=109$; type $2, n=256)$ ]. In total, $39.3 \%$ of respondents had type 1 diabetes, and $60.7 \%$ had type 2 diabetes. Descriptive and health characteristics by diabetes type are shown in Table 1 . The sample was predominantly white and just over half male, and more than half of participants reported being married/partnered and employed. As expected, respondents with type 1 diabetes were significantly younger, and had a significantly younger age at diagnosis and when they first started insulin therapy compared with those with type 2 diabetes.

More than half of the respondents reported that their diabetes was "well" or "very well" controlled (56.2\%) and that their general health status was "good," "very good" or "excellent" (57.3\%). Compared with respondents with type 2 diabetes, respondents with type 1 diabetes were significantly more likely to report that their diabetes was "well" or "very well" controlled (66.3\% vs. $49.6 \% ; p<0.001)$ and that they were in "good," "very good" or "excellent" health $(69.1 \%$ vs. $49.6 \%$; $p<0.001)$. Concerning diet and exercise patterns, respondents had skipped an average of 1.4 meals in the previous week, and over three-quarters of respondents reported eating out in the past week. Respondents with type 1 diabetes reported significantly more hours undertaking moderate and hard physical exercise in the past week compared with those with type 2 diabetes (moderate: 4.0 vs. $3.1 \mathrm{~h}$; $p<0.05$; hard: 2.0 vs. $1.1 \mathrm{~h} ; p<0.001)$.

\section{Out-of-range Post-Prandial Glucose in the Previous Week}

Two-thirds of respondents (66.3\%) indicated experiencing some level of difficulty with out-of-range PPG in the past week, with $7.0 \%$ 
Table 1 Patient demographic and diabetes/health characteristics by diabetes type

\begin{tabular}{|c|c|c|c|}
\hline & Type $1(n=356)$ & Type $2(n=550)$ & Total $(n=906)$ \\
\hline \multicolumn{4}{|l|}{ Patient characteristics } \\
\hline Age (years), mean $(\mathrm{SD})^{* * *}$ & $37.4(14.4)$ & $47.4(16.2)$ & $43.5(16.2)$ \\
\hline Male, $n(\%)$ & $196(55.1)$ & $316(57.5)$ & $512(56.5)$ \\
\hline White Caucasian, $n(\%)$ & $320(89.9)$ & $495(90.0)$ & $815(90.0)$ \\
\hline Married or partnered, $n(\%)$ & $197(55.3)$ & $337(61.3)$ & $534(58.9)$ \\
\hline College/grad school educated, $n(\%)^{*}$ & $159(44.7)$ & $200(36.4)$ & $359(39.6)$ \\
\hline Employed (yes), $n(\%)^{* * *}$ & $231(64.9)$ & $288(52.4)$ & $519(57.3)$ \\
\hline Work hours per week, mean $(S D)^{a}$ & $35.5(13.1)$ & $35.0(10.6)$ & $35.2(11.7)$ \\
\hline \multicolumn{4}{|l|}{ Diabetes/health characteristics } \\
\hline Age (years) at diagnosis, mean $(\mathrm{SD})^{* * *}$ & $20.2(12.1)$ & $35.6(14.0)$ & $29.6(15.3)$ \\
\hline Age (years) started insulin, mean $(\mathrm{SD})^{* * *}$ & $20.9(12.4)$ & $39.6(16.0)$ & $32.2(17.3)$ \\
\hline \multicolumn{4}{|l|}{ Insulin method, $n(\%)^{* * *}$} \\
\hline Syringe & $111(31.2)$ & $187(34.0)$ & $298(32.9)$ \\
\hline Prefilled pen & $173(48.6)$ & $314(57.1)$ & $487(53.8)$ \\
\hline Insulin pump & $72(20.2)$ & $49(8.9)$ & $121(13.4)$ \\
\hline Diabetes well/very well controlled, $n(\%)^{* * *}$ & $236(66.3)$ & $273(49.6)$ & $509(56.2)$ \\
\hline Health status good/very good/excellent, $n(\%)^{* * *}$ & $246(69.1)$ & $273(49.6)$ & $519(57.3)$ \\
\hline \multicolumn{4}{|l|}{ Diet and exercise habits } \\
\hline Number of skipped meals past week, mean (SD) & $1.3(2.2)$ & $1.4(2.0)$ & $1.4(2.1)$ \\
\hline Ate at restaurant in past week (yes), $n$ (\%) & $175(49.2)$ & $302(54.9)$ & $477(52.6)$ \\
\hline Ate takeout in past week (yes), $n$ (\%) & $125(35.1)$ & $205(37.3)$ & $330(36.4)$ \\
\hline Ate at friend's home in past week (yes), $n(\%)$ & $166(46.6)$ & $221(40.2)$ & $387(42.7)$ \\
\hline Any eating out in past week (yes), $n(\%)$ & $267(75.0)$ & $427(77.6)$ & $694(76.6)$ \\
\hline Light physical activity past week, mean hours (SD) & $7.5(7.9)$ & $6.9(7.2)$ & $7.2(7.5)$ \\
\hline Moderate physical activity past week, mean hours $(\mathrm{SD})^{*}$ & $4.0(6.4)$ & $3.1(4.3)$ & $3.5(5.2)$ \\
\hline Hard physical activity past week, mean hours $(\mathrm{SD})^{* * *}$ & $2.0(3.8)$ & $1.1(2.4)$ & $1.5(3.0)$ \\
\hline Physical activity at work (yes), $n(\%)^{a}$ & $45(19.5)$ & $63(21.9)$ & $108(20.8)$ \\
\hline
\end{tabular}

Chi-square/ $t$ tests indicate significant differences by diabetes type, ${ }^{*} p<0.05 ;{ }^{* * *} p<0.001$

${ }^{a}$ Restricted to employed respondents, $n=519$

reporting that it was "very" or "extremely" difficult, and 59.3\% reporting that it was "a little" or "somewhat" difficult to get their BG stable after eating. Difficulty with out-of-range PPG in the past week did not differ significantly by diabetes type. 


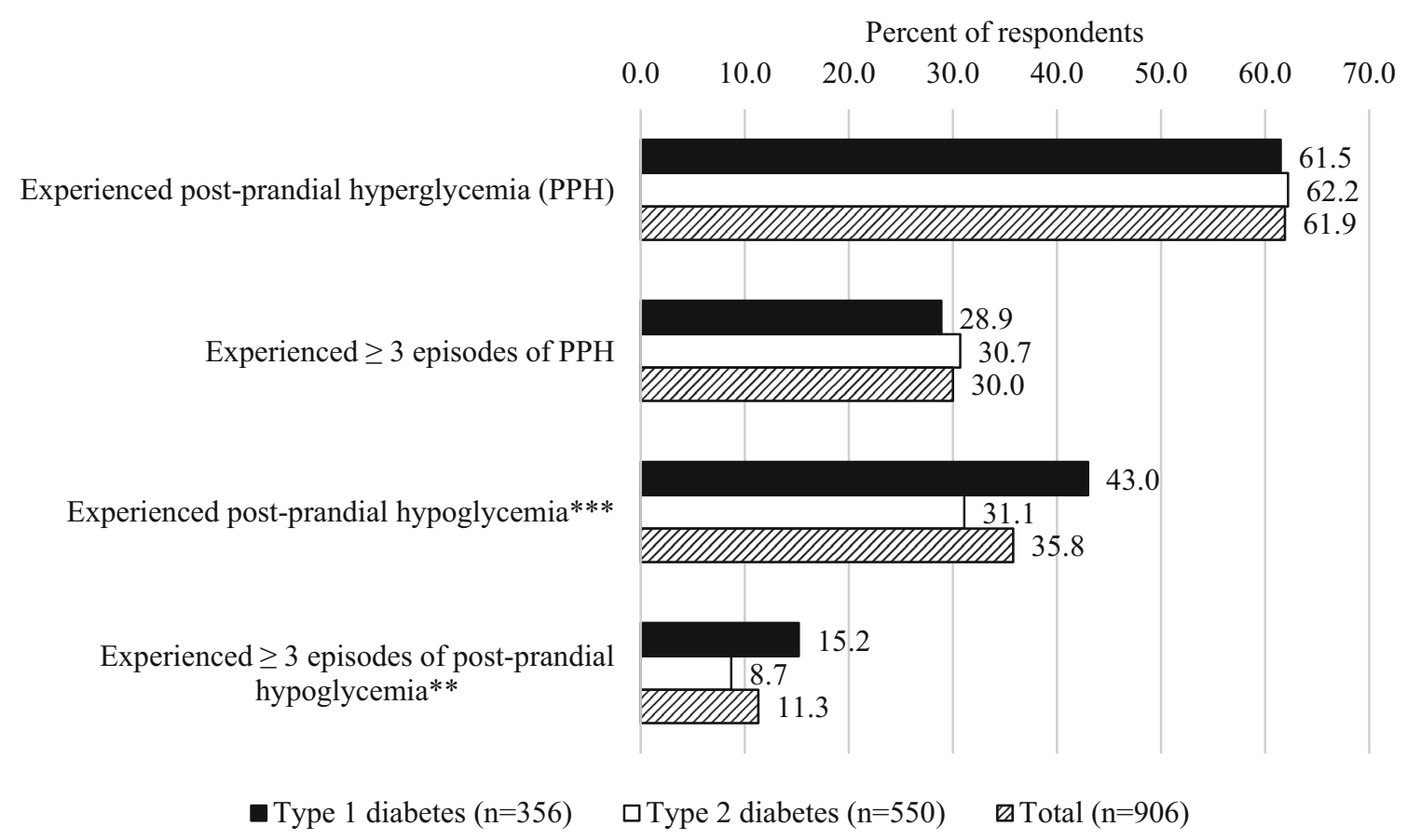

Fig. 1 Out-of-range post-prandial blood glucose in the past week ( 7 days) by diabetes type. Responses were not mutually exclusive. Chi-square tests indicate significant differences by diabetes type, ${ }^{* *} p<0.01 ;{ }^{* * *} p<0.001$. PPH post-prandial hyperglycemia

As shown in Fig. 1, 61.9\% of respondents indicated that they experienced PPH in the past week, $30.0 \%$ experiencing three or more episodes in the past week. A total of $35.8 \%$ of respondents experienced post-prandial hypoglycemia in the past week, with $11.3 \%$ experiencing three or more episodes in the past week. Respondents reported an average of 1.7 episodes of PPH over the course of the week and an average of 0.6 episodes of post-prandial hypoglycemia during the last week.

\section{Respondent Experiences and Management of PPH}

Respondents were asked to report on their most recent episode of out-of-range PPG (Table 2); $52.3 \%$ indicated that their most recent experience was $\mathrm{PPH}$, while $28.4 \%$ indicated it was post-prandial hypoglycemia, and 19.3\% were unsure. The average length of time since this recent episode was 7.5 days. Respondents whose last episode of out-of-range PPG was PPH $(n=474)$ reported additional information on this recent experience of PPH (Table 2). Most respondents indicated that they knew they were experiencing PPH because they tested their BG (64.8\%) and/or "just didn't feel right" (51.9\%). Respondents who usually measured their BG after meals with bolus insulin $(n=353)$ reported testing their BG an average of 92.4 min after meals in the past week.

The most frequent (non-mutually exclusive) reasons that respondents gave for $\mathrm{PPH}$ were: ate more fat/sugar than estimated $(31.2 \%)$, ate more than they calculated their bolus insulin dose for $(30.4 \%)$, forgot to take their bolus insulin dose (17.5\%), calculated their bolus dose incorrectly (16.7\%) and/or took too little bolus insulin (15.2\%). The most frequently reported 
Table 2 Respondents' most recent experiences of post-prandial hyperglycemia by diabetes type

\begin{tabular}{lccc}
\hline $\boldsymbol{n}(\mathbf{\%})^{\mathbf{a}}$ & Type 1 $(n=\mathbf{1 8 7})$ & Type 2 $(\boldsymbol{n}=\mathbf{2 8 7})$ & Total $(\boldsymbol{n}=\mathbf{4}$ \\
\hline How respondent knew episode was PPH & & & \\
Tested BG & $116(62.0)$ & $191(66.6)$ & $307(64.8)$ \\
Just didn't feel right & $100(53.5)$ & $146(50.9)$ & $246(51.9)$ \\
Frequent urination & $18(9.6)$ & $43(15.0)$ & $61(12.9)$ \\
Other & $9(4.8)$ & $21(7.3)$ & $30(6.3)$
\end{tabular}

Top 6 reasons given for $\mathrm{PPH}$ developing

Ate more fat/sugar than estimated

$55(29.4)$
$56(29.9)$
$30(16.0)$
$37(19.8)$
$36(19.3)$
$19(10.2)$

$93(32.4)$

148 (31.2)

Ate more than calculated bolus dose for

88 (30.7)

$144(30.4)$

Forgot to take bolus dose

$53(18.5)$

$83(17.5)$

Calculated bolus dose incorrectly

$42(14.6)$

$79(16.7)$

Took too little bolus insulin*

$36(12.5)$

$72(15.2)$

Because of stress

39 (13.6)

$58(12.2)$

Top 5 situations/factors contributing to $\mathrm{PPH}$

$\begin{array}{ll}\text { Stress* }^{* *} & 36(19.3) \\ \text { Eating at a restaurant } & 43(23.0) \\ \text { Being busy } & 34(18.2) \\ \text { Feeling tired } & 24(12.8) \\ \text { I do not know* } & 37(19.8)\end{array}$

$94(32.8)$

$130(27.4)$

$3(23.0)$

$75(26.1)$

$118(24.9)$

Being busy

$66(23.0)$

$100(21.1)$

$4(12.8)$

67 (23.3)

$91(19.2)$

34 (11.8)

$71(15.0)$

Corrective action taken following PPH

Tested BG and took bolus based on reading ${ }^{* * *} \quad 134$ (71.7)

$60(55.7)$

$294(62.0)$

Ate less/more carefully next meal/snack* ${ }^{*}$

$22(11.8)$

$67(23.3)$

89 (18.8)

Waited, took bolus dose next time I ate

$17(9.1)$

40 (13.9)

$57(12.0)$

Took bolus dose without testing BG

$17(9.1)$

39 (13.6)

$56(11.8)$

Skipped next meal/snack

$8(4.3)$

$14(4.9)$

$22(4.6)$

Other

$6(3.2)$

$15(5.2)$

$21(4.4)$

Total took bolus dose**

$147(78.6)$

$191(66.6)$

$338(71.3)$

Chi-square tests indicate significant differences by diabetes type, ${ }^{*} p<0.05 ;{ }^{* *} p<0.01 ;{ }^{* * *} p<0.001$ $P P H$ post-prandial hyperglycemia, $B G$ blood glucose

${ }^{a}$ Responses not mutually exclusive

(non-mutually exclusive) situations which contributed to PPH were: stress (27.4\%), eating at a restaurant $(24.9 \%)$, being busy $(21.1 \%)$ and/ or feeling tired (19.2\%).
The most frequently reported (non-mutually exclusive) corrective actions taken by respondents following PPH were: tested BG and took bolus insulin based on the reading 
(62.0\%) and/or ate less/more carefully for the next meal or snack (18.8\%). Of note, $12.0 \%$ of respondents reported that they waited and took a bolus dose with their next meal/snack, and $11.8 \%$ reported taking a bolus dose without testing their BG.

Experiences of PPH differed somewhat by diabetes type. Patients with type 1 diabetes, when compared with those with type 2 diabetes, were significantly more likely to indicate that they took too little bolus insulin as a reason for PPH (19.3\% vs. $12.5 \%$; $p<0.05)$. Patients with type 2 diabetes were significantly more likely to indicate both "stress" (32.8\% vs. $19.3 \%, p<0.01)$ and "feeling tired" (23.3\% vs. $12.8 \% ; \quad p<0.01)$ as contributing factors/ situations for $\mathrm{PPH}$ compared with patients with type 1 diabetes. In terms of corrective action following $\mathrm{PPH}$, patients with type 1 diabetes were significantly more likely to indicate that they tested BG and took a bolus dose based on the reading $(71.1 \%$ vs. $55.7 \%$ for type 2 diabetes; $p<0.001$ ), whereas those with type 2 diabetes were significantly more likely to indicate that they ate less/more carefully at the next meal/snack $(23.3 \%$ vs. $11.8 \%$ for type 1 diabetes; $p<0.01)$.

\section{Comparison of Respondents Who Did and Did Not Experience PPH}

Respondents who did and did not experience $\mathrm{PPH}$ in the past week were compared in terms of diabetes and health characteristics, as well as emotions when taking bolus insulin and diabetes-related concerns. Regarding diabetes characteristics, the method of insulin administration differed significantly between respondents who experienced $\mathrm{PPH}$ and those who did not $(p<0.001)$; while both groups were approximately equally likely to use a syringe (32.3\% and 33.9\%, respectively), respondents who experienced PPH were less likely to use a prefilled pen (48.8\% vs. $61.7 \%$ ) and somewhat more likely to use an insulin pump (18.9\% vs. 13.4\%). Further, respondents who experienced PPH were significantly less likely to report that their diabetes was "well" or "very well" controlled ( $49.9 \%$ vs. $66.4 \% ; p<0.001)$ or that their general health status was "good," "very good" or "excellent" (54.4\% vs. $62.0 \%$; $p<0.05)$ compared with those who did not experience PPH.

In terms of eating habits, respondents who experienced $\mathrm{PPH}$ in the past week reported skipping a significantly greater number of meals in the past week (1.6 vs. $1.0 ; p<0.001)$ and were significantly more likely to have eaten out at a restaurant $(57.2 \%$ vs. $45.2 \%$; $p<0.001)$, eaten takeout food $(40.1 \%$ vs. $30.4 \% ; p<0.01)$, and/or eaten at a friend's home $(46.3 \%$ vs. $36.8 \% ; p<0.01)$ than those who did not experience PPH. Reported physical activity did not differ significantly between those who had and had not experienced PPH in the past week.

Significant differences in emotions when taking bolus insulin were evident, with those who experienced $\mathrm{PPH}$ in the past week significantly more likely to report feeling very/ extremely "grateful" (31.6\% vs. $23.2 \%$; $p<0.01)$, "worried" $(10.2 \%$ vs. $5.2 \% ; p<0.01)$, "guilty" (9.8\% vs. $4.3 \%$; $p<0.01)$, "discouraged" $(11.8 \%$ vs. $5.2 \% ; p<0.01)$, "frustrated" $(12.5 \%$ vs. $6.4 \% ; p<0.01)$, "embarrassed" $(8.2 \%$ vs. $4.1 \% ; p<0.05)$, and "angry" (9.3\% vs. $4.6 \%$, $p<0.05)$ when taking bolus insulin compared with those who did not experience $\mathrm{PPH}$ (Table 3). Additionally, those who experienced $\mathrm{PPH}$ in the past week were significantly less likely to report that they were very/extremely confident in calculating their bolus doses accurately $\quad(52.9 \% \quad$ vs. $\quad 67.5 \% ; \quad p<0.001)$ compared with those who did not experience $\mathrm{PPH}$. Regarding diabetes-related concerns, those 
who experienced PPH were significantly more likely to indicate being very/extremely worried about future diabetes complications (45.6\% vs. $25.8 \% ; \quad p<0.001)$, whether insulin was controlling their diabetes $(30.1 \%$ vs. $13.0 \%$; $p<0.001)$, high blood sugar events $(30.7 \%$ vs. 9.6\%; $p<0.001)$, low blood sugar events $(24.8 \%$ vs. $11.3 \% ; p<0.001)$, whether they were calculating bolus insulin doses correctly (21.6\% vs. $10.7 \% ; p<0.001)$, and using their insulin devices $(9.8 \%$ vs. $4.1 \% ; p<0.01)$.
Associations between PPH and respondent emotions when taking bolus insulin differed somewhat by diabetes type. The positive relationships between PPH and some emotions when taking bolus doses were significant among respondents with type 1 diabetes, but not among those with type 2 diabetes, including feeling very/extremely "grateful" $(p<0.05)$ and "embarrassed" $(p<0.05)$. In addition, the associations between experience of $\mathrm{PPH}$ and a number of emotions when taking bolus insulin

Table 3 Respondents' feelings about bolus insulin and general worries related to diabetes according to whether they had experienced post-prandial hyperglycemia in the past week ( 7 days)

\begin{tabular}{|c|c|c|c|}
\hline$n(\%)^{\mathrm{a}}$ & $\begin{array}{l}\text { PPH in past } \\
\text { week }(n=561)\end{array}$ & $\begin{array}{l}\text { No PPH in past } \\
\text { week }(n=345)\end{array}$ & $\begin{array}{l}\text { Total } \\
(n=906)\end{array}$ \\
\hline \multicolumn{4}{|l|}{ When you take your bolus insulin doses, do you feel very/extremely? } \\
\hline Grateful $^{* *}$ & $177(31.6)$ & $80(23.2)$ & $257(28.4)$ \\
\hline Empowered & $122(21.7)$ & $66(19.1)$ & $188(20.8)$ \\
\hline Frustrated ${ }^{* *}$ & $70(12.5)$ & $22(6.4)$ & $92(10.2)$ \\
\hline Discouraged $^{* *}$ & $66(11.8)$ & $18(5.2)$ & $84(9.3)$ \\
\hline Worried ${ }^{* *}$ & $57(10.2)$ & $18(5.2)$ & $75(8.3)$ \\
\hline Guilty** & $55(9.8)$ & $15(4.3)$ & $70(7.7)$ \\
\hline Angry* & $52(9.3)$ & $16(4.6)$ & $68(7.5)$ \\
\hline Embarrassed* & $46(8.2)$ & $14(4.1)$ & $60(6.6)$ \\
\hline $\begin{array}{l}\text { Are you very/extremely confident that you really know how to } \\
\text { accurately calculate your bolus doses? } \text { ? }^{* *}\end{array}$ & $297(52.9)$ & $233(67.5)$ & $530(58.5)$ \\
\hline \multicolumn{4}{|l|}{ In general, are you very/extremely worried about? } \\
\hline Future diabetes complications $s^{* * *}$ & $256(45.6)$ & $89(25.8)$ & $345(38.1)$ \\
\hline If your insulin is controlling your diabetes ${ }^{* * *}$ & $169(30.1)$ & $45(13.0)$ & $214(23.6)$ \\
\hline High blood sugar events ${ }^{* * *}$ & $172(30.7)$ & $33(9.6)$ & $205(22.6)$ \\
\hline Low blood sugar events ${ }^{* * *}$ & $139(24.8)$ & $39(11.3)$ & $178(19.6)$ \\
\hline If you are calculating insulin doses correctly $y^{* * *}$ & $121(21.6)$ & $37(10.7)$ & $158(17.4)$ \\
\hline Problems contacting your healthcare provider & $54(9.6)$ & $21(6.1)$ & $75(8.3)$ \\
\hline Using your insulin device** & $55(9.8)$ & $14(4.1)$ & $69(7.6)$ \\
\hline
\end{tabular}

Chi-square tests indicate significant differences by experience of PPH in past week, ${ }^{*} p<0.05 ;{ }^{* *} p<0.01 ;{ }^{* * *} p<0.001$ $P P H$ post-prandial hyperglycemia

${ }^{a}$ Denotes proportion of patients answering yes 
were significant among respondents with type 2 diabetes, but not among those with type 1 diabetes, including feeling very/extremely "empowered" $(p<0.05)$, "worried" $(p<0.01)$, "frustrated" $(p<0.01)$ or "angry" $(p<0.05)$ when taking bolus insulin, as well as confidence in calculating their bolus insulin dose $(p<0.001)$. Additionally, the relationships between PPH and some other diabetes-related concerns were significant among respondents with type 2 diabetes, but not among those with type 1 diabetes, including being very/extremely confident in calculating the bolus dose $(p<0.001)$ and being very/extremely worried about hypoglycemic events $(p<0.001)$, using the insulin device $(p<0.01)$, future diabetes complications $(p<0.001)$, and whether or not they were calculating insulin doses correctly $(p<0.001)$.

\section{DISCUSSION}

Addressing PPH may be critical for achieving better BG control, thereby reducing the risks of diabetes-related complications and mortality [8-17]. Consistent with prior research [2, 7], this study shows that PPH occurs frequently among patients with type 1 or type 2 diabetes. This study adds to previous research by examining how patients with diabetes experience $\mathrm{PPH}$ in their everyday lives, including causes and contributing factors to $\mathrm{PPH}$, as well as emotions surrounding bolus insulin use and diabetes-related concerns associated with PPH. Results indicate that eating more than estimated and over-eating with respect to the calculated bolus insulin dose are frequently reported reasons for $\mathrm{PPH}$, and that a number of factors in one's daily life, including stress, eating out, being busy, and feeling tired, may also contribute to $\mathrm{PPH}$.
Experience of PPH was associated with more negative emotions when taking bolus insulin doses, less confidence in calculating bolus doses accurately, and greater diabetes-related concerns. Thus, the findings suggest that PPH may be associated with an emotional burden related to diabetes self-management. The development of PPH also appears to be associated with particular eating habits, for example, eating out and skipped meals. Overall, the findings illuminate how patients with diabetes experience PPH in their day-to-day lives and add to previous literature indicating that the experience of hyperglycemia has negative implications for quality of life and well-being among patients with diabetes $[22,23]$.

The findings from this web-based survey have implications for both clinicians and patients. Patient education and support programs to help diabetic patients manage their eating habits and lifestyle issues that may contribute to PPH could help improve BG control and reduce the number of episodes of $\mathrm{PPH}$. The provision of continuing education for patients on the use of bolus insulin could also help improve diabetes self-management and increase patients' confidence when calculating their bolus insulin dose. Counseling and patient support groups may also help mitigate negative emotions surrounding bolus insulin use and diabetes-related concerns associated with PPH.

The results also suggest some differences in the experience of PPH according to diabetes type. Patients with type 2 diabetes may be particularly vulnerable to stress and tiredness as contributing factors to PPH and are less likely to measure their BG following $\mathrm{PPH}$, compared with type 1 diabetic patients. In terms of emotions when using bolus insulin and diabetes-related concerns associated with $\mathrm{PPH}$, it is difficult to draw general conclusions about 
differences according to diabetes type as the results were somewhat mixed. Some non-significant findings among respondents with type 1 diabetes may be due to the smaller sample size relative to respondents with type 2 diabetes. Additional research is needed to better understand the influence of diabetes type on emotions.

Finally, this study has some limitations which should be considered. First, all survey data were self-reported. As with any self-reported survey, reporting accuracy may be a concern, as recall bias may have impacted the results. Unfortunately, data on actual BG measurements were not available to confirm respondents' experiences of PPH or the severity of episodes. Research, however, demonstrates that the recall of non-severe hypoglycemic episodes of up to 1 week may be considered generally accurate [24]. Furthermore, participants in the focus groups during the survey development phase of the study indicated that the recall periods used for the survey were appropriate. Additionally, physician-confirmed diagnosis of diabetes was not possible, so some respondents may have reported a diabetes diagnosis, diabetes type, and/or treatment with bolus insulin therapy inaccurately. It is unlikely, however, that such respondents were large enough in numbers to influence study results. Lastly, as with all studies that use internet-based surveys, data may be affected by selection bias related to respondent participation, as only literate respondents with computer and internet access could participate. However, in the three countries studied, the literacy rates and proportion of internet users are both quite high. In the UK, for example, the basic literacy rate of adults aged 15 years and older is $99 \%$ [25], and approximately $83 \%$ of households had internet access in 2013 [26].

\section{CONCLUSION}

In conclusion, these findings shed much needed light on how patients with type 1 and type 2 diabetes experience PPH and how $\mathrm{PPH}$ contributes to the complexities of diabetes self-management. Clinicians and diabetes educators should be aware of the eating habits and lifestyle issues that may contribute to $\mathrm{PPH}$, including stress, being busy, eating out, and skipped meals, so that they can educate patients with diabetes on the serious consequences of $\mathrm{PPH}$ and help them develop targeted self-management strategies to reduce PPH [2]. Clinicians should also be mindful that experience of PPH may contribute to negative emotions when using bolus insulin, as well as greater diabetes-related concerns. Additional patient education and support programs may help mitigate such negative emotions and concerns. Future research should focus on improving diabetes self-management and treatments to reduce the incidence of $\mathrm{PPH}$, as well as explore broader and longer term impacts of PPH. Further exploration of country and cultural differences in the experience of $\mathrm{PPH}$ would be of interest.

\section{ACKNOWLEDGMENTS}

Sponsorship and article processing charges for this study were funded by Novo Nordisk. All named authors meet the International Committee of Medical Journal Editors (ICMJE) criteria for authorship for this manuscript, take responsibility for the integrity of the work as a whole, and have given final approval to the version to be published. No medical writing assistance was provided for this study. 
Disclosures. Meryl Brod is a paid consultant to the pharmaceutical industry, including Novo Nordisk. Kathryn M. Pfeiffer is a paid consultant to the pharmaceutical industry, including Novo Nordisk. Annie Nikolajsen is an employee of Novo Nordisk A/S. James Weatherall is an employee of Novo Nordisk, Inc.

Compliance with Ethics Guidelines. Prior to commencement, the study received ethics approval from Copernicus Group Institutional Review Board (Tracking \# TBG1-11-116). All procedures followed were in accordance with the ethical standards of the responsible committee on human experimentation (institutional and national) and with the Helsinki Declaration of 1964, as revised in 2013. Informed consent was obtained from all participants for being included in the study.

Open Access. This article is distributed under the terms of the Creative Commons Attribution-NonCommercial 4.0 International License (http://creativecommons.org/licenses/ by-nc/4.0/), which permits any noncommercial use, distribution, and reproduction in any medium, provided you give appropriate credit to the original author(s) and the source, provide a link to the Creative Commons license, and indicate if changes were made.

\section{REFERENCES}

1. American Diabetes Association. Standards of medical care in diabetes-2014. Diabetes Care. 2014;37:S14-80.

2. International Diabetes Federation. Guideline for management of postmeal glucose in diabetes. 2011. http://www.idf.org/2011-guideline-managementpostmeal-glucose-diabetes. Accessed 27 Mar 2014.

3. Nathan DM, DCCT/EDIC Research Group. The diabetes control and complications trial/ epidemiology of diabetes interventions and complications study at 30 years: overview. Diabetes Care. 2014;37:9-16.

4. Stratton IM, Adler AI, Neil HA, et al. Association of glycaemia with macrovascular and microvascular complications of type 2 diabetes (UKPDS 35): prospective observational study. BMJ. 2000;321:405-12.

5. Ceriello A. The glucose triad and its role in comprehensive glycaemic control: current status, future management. Int $\mathrm{J}$ Clin Pract. 2010;64:1705-11.

6. Lebovitz HE, Austin MM, Blonde L, et al. ACE/AACE consensus conference on the implementation of outpatient management of diabetes mellitus: consensus conference recommendations. Endoc Pract. 2006;12(Supplement 1):6-12.

7. Bonora E, Corrao G, Bagnardi V, et al. Prevalence and correlates of post-prandial hyperglycaemia in a large sample of patients with type 2 diabetes mellitus. Diabetologia. 2006;49:846-54.

8. Aryangat AV, Gerich JE. Type 2 diabetes: postprandial hyperglycemia and increased cardiovascular risk. Vasc Health Risk Manag. 2010;6:145-55.

9. Ceriello A. Cardiovascular effects of acute hyperglycaemia: pathophysiological underpinnings. Diabetes Vasc Dis Res. 2008;5:260-8.

10. Standl E, Schnell O, Ceriello A. Postprandial hyperglycemia and glycemic variability should we care? Diabetes Care. 2011;34:S120-7.

11. Cavalot F, Pagliarino A, Valle $M$, et al. Postprandial blood glucose predicts cardiovascular events and all-cause mortality in type 2 diabetes in a 14-year follow-up: lessons from the San Luigi Gonzaga Diabetes Study. Diabetes Care. 2011;34:2237-43.

12. Cavalot F, Petrelli A, Traversa M, et al. Postprandial blood glucose is a stronger predictor of cardiovascular events than fasting blood glucose in type 2 diabetes mellitus, particularly in women: lessons from the San Luigi Gonzaga Diabetes Study. J Clin Endocrinol Metab. 2006;91:813-9.

13. Barr EL, Zimmet PZ, Welborn TA, et al. Risk of cardiovascular and all-cause mortality in individuals with diabetes mellitus, impaired fasting glucose, and impaired glucose tolerance: the Australian Diabetes, Obesity, and Lifestyle Study (AusDiab). Circulation. 2007;116:151-7.

14. Lowe LP, Liu K, Greenland P, Metzger BE, Dyer AR, Stamler J. Diabetes, asymptomatic hyperglycemia, and 22-year mortality in black and white men: the 
Chicago Heart Association Detection Project in Industry Study. Diabetes Care. 1997;20:163-9.

15. Nakagami T. Hyperglycaemia and mortality from all causes and from cardiovascular disease in five populations of Asian origin. Diabetologia. 2004;47:385-94.

16. Saydah SH, Miret M, Sung J, Varas C, Gause D, Brancati FL. Postchallenge hyperglycemia and mortality in a national sample of US adults. Diabetes Care. 2001;24:1397-402.

17. Gapstur SM, Gann PH, Lowe W, Liu K, Colangelo L, Dyer A. Abnormal glucose metabolism and pancreatic cancer mortality. JAMA. 2000;283:2552-8.

18. Rizzo MR, Marfella R, Barbieri $M$, et al. Relationships between daily acute glucose fluctuations and cognitive performance among aged type 2 diabetic patients. Diabetes Care. 2010;33:2169-74.

19. Brod M, Nikolajsen A, Weatherall J, Pfeiffer KM. The economic burden of post-prandial hyperglycemia (PPH) among people with Type 1 and Type 2 diabetes in three countries. Diabetes Ther. 2016;7:75-90.

20. Lawton JD, Rankin D, Cooke D, et al. Patients' experiences of adjusting insulin doses when implementing flexible intensive insulin therapy: a longitudinal, qualitative investigation. Diabetes Res Clin Pract. 2012;98:236-42.
21. Charmaz K. Premises, principles, and practices in qualitative research: revisiting the foundations. Qual Health Res. 2004;14:976-93.

22. Tabaei BP, Shill-Novak J, Brandle M, Burke R, Kaplan RM, Herman WH. Glycemia and the quality of well-being in patients with diabetes. Qual Life Res. 2004;13:1153-61.

23. Kleefstra N, Ubink-Veltmaat LJ, Houweling ST, Groenier KH, Meyboom-de Jong B, Bilo HJ. Cross-sectional relationship between glycaemic control, hyperglycaemic symptoms and quality of life in type 2 diabetes (ZODIAC-2). Neth J Med. 2005;63:215-21.

24. Pramming S, Thorsteinsson B, Bendtson I, et al. Symptomatic hypoglycaemia in 411 type 1 diabetic patients. Diabet Med. 1991;8:217-22.

25. Central Intelligence Agency. The World Factbook: United Kingdom. Washington, DC: CIA; 2014. https://www.cia.gov/library/publications/the-worldfactbook/geos/uk.html. Accessed 17 July 2014.

26. U.K. Office for National Statistics. Statistical bulletin: internet access-households and individuals, 2013. UK. http://www.ons.gov. uk/ons/rel/rdit2/internet-access-households-andindividuals/2013/stb-ia-2013.html\#tab-Computerand-Internet-use. Accessed 17 July 2014. 